\title{
Ocorrência de Rhopalopsyllus lutzi lutzi (Baker) (Siphonaptera, Rhopalopsyllidae) em Canis familiaris (Linnaeus) de zona rural do município de Piraí, Rio de Janeiro, Brasil
}

\author{
Alessandra Scofield ${ }^{1}$, Maria Dalila Forlano Riera ${ }^{1}$, Carina Elisei ${ }^{1}$, Carlos Luiz Massard \\ \& Pedro Marcos Linardi ${ }^{2}$
}

${ }^{1}$ Curso de Pós-Graduação em Ciências Veterinárias, Departamento de Parasitologia Animal, Instituto de Veterinária, Universidade Federal Rural do Rio de Janeiro. 23890-000 Seropédica KM 7-RJ. lecascofield@hotmail.com

${ }^{2}$ Departamento de Parasitologia, Instituto de Ciências Biológicas, Universidade Federal de Minas Gerais Caixa Postal 486, $30161-970$

Belo Horizonte-MG. linardi@icb.ufmg.br. Bolsista do CNPq.

\begin{abstract}
Occurrence of Rhopalopsyllus lutzi lutzi (Baker) (Siphonaptera, Rhopalopsyllidae) on Canis familiaris (Linnaeus) from rural areas of the county of Piraí, State of Rio de Janeiro, Brazil. From June/2001 to November/2003, 51 fleas were collected on eight dogs from two rural properties of the county of Piraí, State of Rio de Janeiro. After preservation in $70 \%$ ethanol, the fleas were counted and sexed in the Laboratório de Parasitologia Animal da Universidade Federal Rural do Rio de Janeiro. The samples were identificated as Rhopalopsyllus lutzi lutzi (Baker, 1904) (male $=18$ and female $=33$ ). For the first time $R$. lutzi lutzi was found paraziting dogs in rural areas of the State of Rio de Janeiro.
\end{abstract}

KEYwords. Dogs; fleas; Rhopalopsyllidae; Rhopalopsyllus lutzi lutzi; Siphonaptera.

Resumo. Ocorrência de Rhopalopsyllus lutzi lutzi (Baker) (Siphonaptera, Rhopalopsyllidae) em Canis familiaris (Linnaeus) de zona rural do município de Piraí, Rio de Janeiro, Brasil. A ocorrência de Rhopalopsyllus lutzi lutzi (Baker) (Siphonaptera, Rhopalopsyllidae) foi assinalada em Canis familiaris (Linnaeus) de áreas rurais do município de Piraí, estado do Rio de Janeiro, Brasil. No período de junho 2001/novembro 2003, 51 sifonápteros foram capturados em oito cães procedentes de duas propriedades rurais do município de Piraí. Os exemplares coletados foram acondicionados em álcool etílico 70\%, levados ao Laboratório de Parasitologia Animal da Universidade Federal Rural do Rio de Janeiro para contagem e sexagem. Os exemplares foram identificados como Rhopalopsyllus lutzi lutzi Baker, 1904 (machos=18 e fêmeas=33). $R$. lutzi lutzi é, pela primeira vez, assinalada em cães domésticos naturalmente infestados em áreas rurais do estado do Rio de Janeiro.

Palavras-Chave. Cães; pulgas; Rhopalopsyllidae; Rhopalopsyllus lutzi lutzi; Siphonaptera.

As pulgas são membros da ordem Siphonaptera e parasitam animais de sangue quente (Beauty \& Marquardt 1996), podendo apresentar especificidade parasitária ou não (Linardi 1985; Soulsby 1987; Kettle 1995).

Estudos realizados no Brasil demonstraram a ocorrência de oito famílias, 20 gêneros e 59 espécies e/ou subespécies distribuídos em quase todo território, sendo encontradas mais freqüentemente as famílias Pulicidae e Rhopalopsyllidae (Linardi \& Guimarães 2000).

A família Rhopalopsyllidae é considerada a mais importante, devido à sua ampla distribuição geográfica, número de táxons e caráter endêmico de diversas espécies (Linardi 1984; Linardi \& Guimarães 2000). Esta família está constituída por duas subfamílias, Rhopalopsyllinae e Parapsyllinae, com apenas a primeira ocorrendo no Brasil (Smit 1987). Ainda que por meio de análises fenética e cladística, oito gêneros sejam reconhecidos entre os Rhopalopsyllinae (Linardi \& Guimarães 1993), cinco foram até o presente, assinalados no Brasil: Rhopalopsyllus Baker, 1905; Polygenis Jordan, 1939; Gephyropsylla Barrera, 1952; Hechtiella Barrera, 1952 e Neotropsylla Linardi \& Guimarães, 1993. Nessa subfamília, as espécies do gênero Rhopalopsyllus apresentam maior diversidade parasitária de hospedeiros (Linardi 1985; Linardi \& Guimarães 2000).
Rhopalopsyllus lutzi (Baker, 1904) é a espécie-tipo do gênero, tendo sido originalmente descrita de Grison (= Galictis vittata Schreber, 1776) do estado de São Paulo. Uma outra espécie próxima, R. cleophontis Rothschild, 1904 foi posteriormente considerada por Jordan \& Rothschild (1923) como subespécie de $R$. lutzi. Atualmente, $R$. lutzi lutzi ocorre no Brasil e Paraguai e $R$. lutzi cleophontis, na Argentina. Infestações por $R$. lutzi lutzi já foram descritas em diferentes ordens de hospedeiros como Marsupialia Ameghino, 1889 (Linardi 1985; Barros-Battesti \& Arzua 1997), Edentata, Rodentia e Carnivora (Linardi 1985), podendo ainda ser encontrada livre em ninhos e habitações (Linardi \& Guimarães 2000).

O presente trabalho teve como objetivo identificar uma espécie de sifonáptero pertencente à família Rhopalopsyllidae infestando cães domésticos, provenientes de zonas rurais do município de Piraí, estado do Rio de Janeiro, Brasil.

\section{MATERIAL E MÉTODOS}

No período de junho 2001/novembro 2003, 51 sifonápteros foram capturados em oito cães (Canis familiaris) procedentes de duas propriedades rurais do município de Piraí, mesorregião do Médio Paraíba, estado do Rio de Janeiro. Esses cães, 


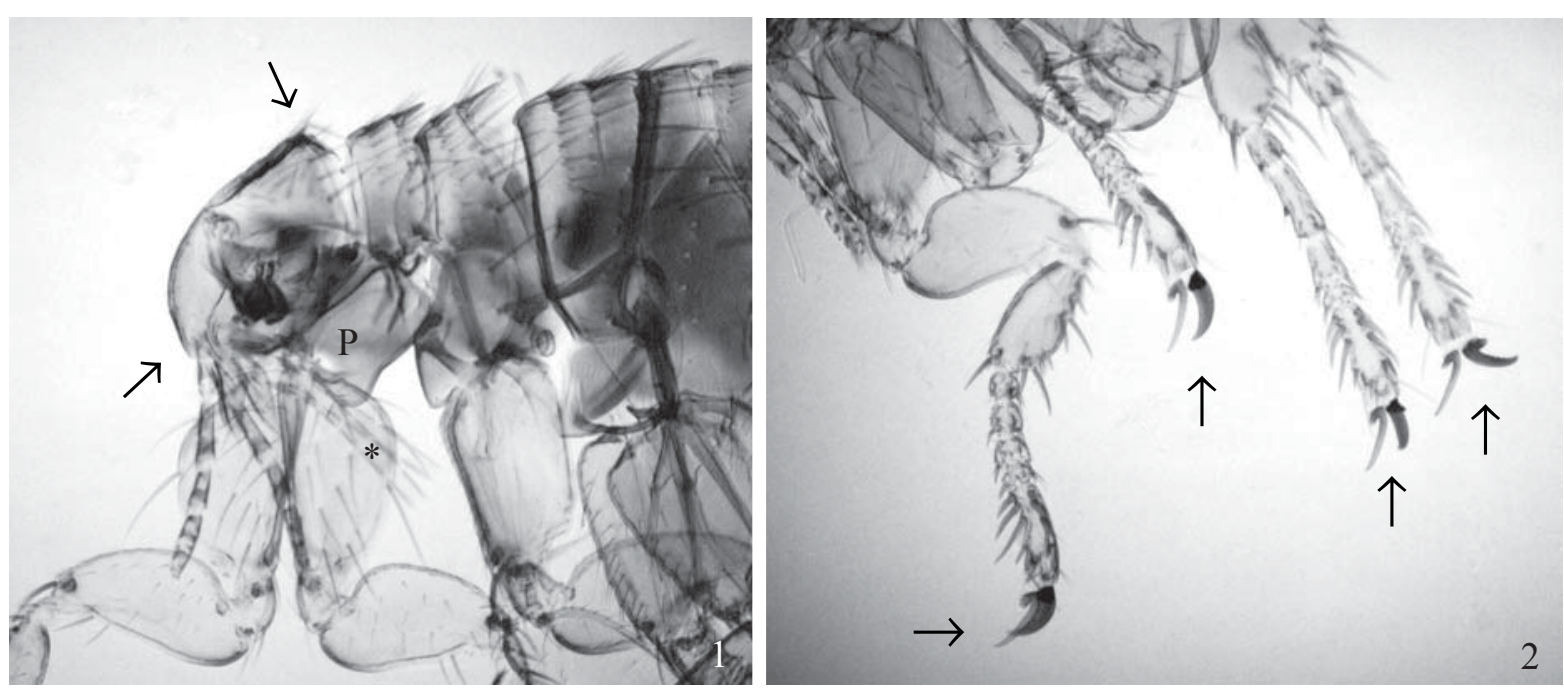

Figs. 1-2. Rhopalopsyllus lutzi lutzi. 1. ausência de ctenídio, forma do prosterno que se projeta entre as coxas (P), palpos labiais com seis ou sete segmentos $(*)$; 2. unhas do primeiro e segundo pares de pernas assimétricas.

mantidos em ambiente domiciliar e peridomiciliar, tinham acesso à mata e freqüentemente capturavam animais silvestres, segundo informações dos proprietários. Após apreensão, os animais foram amordaçados, inspecionados e a captura das pulgas realizada através de catação manual e auxílio de pente fino.

Os exemplares coletados foram acondicionados em recipientes plásticos contendo álcool etílico $70^{\circ}$, sendo transportados ao Laboratório de Parasitologia Animal da Universidade Federal Rural do Rio de Janeiro para contagem e sexagem. Alguns espécimes foram clarificados em potassa fria e montados em bálsamo do Canadá, entre lâminas e lamínulas (Linardi \& Guimarães 2000). Posteriormente, foram fotografados, utilizando-se um microscópio fotônico (Dialux 20 Eb-Leitz) e câmara fotográfica.

Uma amostra dos exemplares foi enviada ao Laboratório de Ectoparasitos do Instituto de Ciências Biológicas da Universidade Federal de Minas Gerais para a identificação e depósito na Coleção de Ectoparasitos do Departamento de Parasitologia da mesma Universidade. Os demais exemplares foram depositados na Coleção Entomológica “Ângelo Moreira da Costa Lima", no Instituto de Biologia da UFRRJ.

\section{RESULTADOS EDISCUSSÃO}

Os 51 sifonápteros capturados (machos $=18$ e fêmeas $=33$ ) foram identificados como $R$. lutzi lutzi. A intensidade média de infestação foi de 6,37. A relação fêmea/macho obtida entre os exemplares capturados foi de 1,83 , sendo um pouco maior que aquela apresentada por Linardi \& Guimarães (2000) e noticiada como de 1,41 entre 58 exemplares examinados, ainda que os dados daqueles autores incluíssem maior número de exemplares capturados livremente ou sobre animais silvestres.

Conforme as figuras apresentadas, as principais características desta espécie são: ausência de ctenídio, forma do prosterno projetando-se entre as coxas, palpos labiais com seis ou sete segmentos (Fig. 1), unhas do primeiro e segundo pares de pernas assimétricas (Fig.2), genitália da fêmea apresentando espermateca com bulga elíptica (Fig.3), e genitália do macho com os segmentos modificados: processo móvel do clasper (telômero) e esternito IX (Fig.4).

Os primeiros exemplares capturados foram identificados no gênero Polygenis Jordan, 1939 (Scofield et al. 2002), devido ao uso incorreto de chave pictórica incluída em Bicho \& Ribeiro (1998). Os resultados ora apresentados confirmam como $R$. lutzi lutzi (Linardi \& Guimarães 2000) os exemplares capturados durante o período de estudo.

Em vários estados brasileiros informações sobre ropalopsilinos são escassas ou inexistentes (Linardi 1987). Registros de $R$. lutzi lutzi infestando diferentes espécies de hospedeiros foram assinalados nos estados de Goiás, Mato Grosso do Sul, Rondônia, Paraná, São Paulo, Minas Gerais e Rio de Janeiro (Linardi \& Guimarães 2000), não relacionando o cão doméstico como hospedeiro.

As ordens Rodentia e Marsupialia são as mais freqüentemente infestadas por Rhopalopsyllus, seguidas por Carnivora, devido aos hábitos predatórios dos seus representantes (Linardi 1985; Linardi \& Guimarães 2000). Infestações por $R$. lutzi lutzi foram registradas nos seguintes hospedeiros brasileiros: rato-do-chão (Akodon serrensis Thomas, 1902) por Barros et al. (1993); cutias (Dasyprocta aguti $=$ Dasyprocta leporina Linnaeus, $1758, D$. azarae Lichtenstein, 1823), tatu (Dasypus novemcinctus Linnaeus, 1758), gambás (Didelphis albiventris Lund, 1840, D. marsupialis Linnaeus, 1758, D. m. aurita $=$ Didelphis aurita Wied-Neuwied, 1826), cuíca (Philander opossum Linnaeus, 1758), tamanduá-mirim (Tamandua tetradactyla Linnaeus, 1758), cachorro-do-mato (Dusicyon thous melanopus $=$ Cerdocyon thous Linnaeus, 1766), jaguatirica (Leopardus pardalis chibigouazou $=$ Leopardus pardalis Linnaeus, 1758), gato-mourisco (Herpailurus yaguarondy Lacépède, 1809), furão (Galictis vittata Schreber, 1776) e quatis (Nasua nasua 

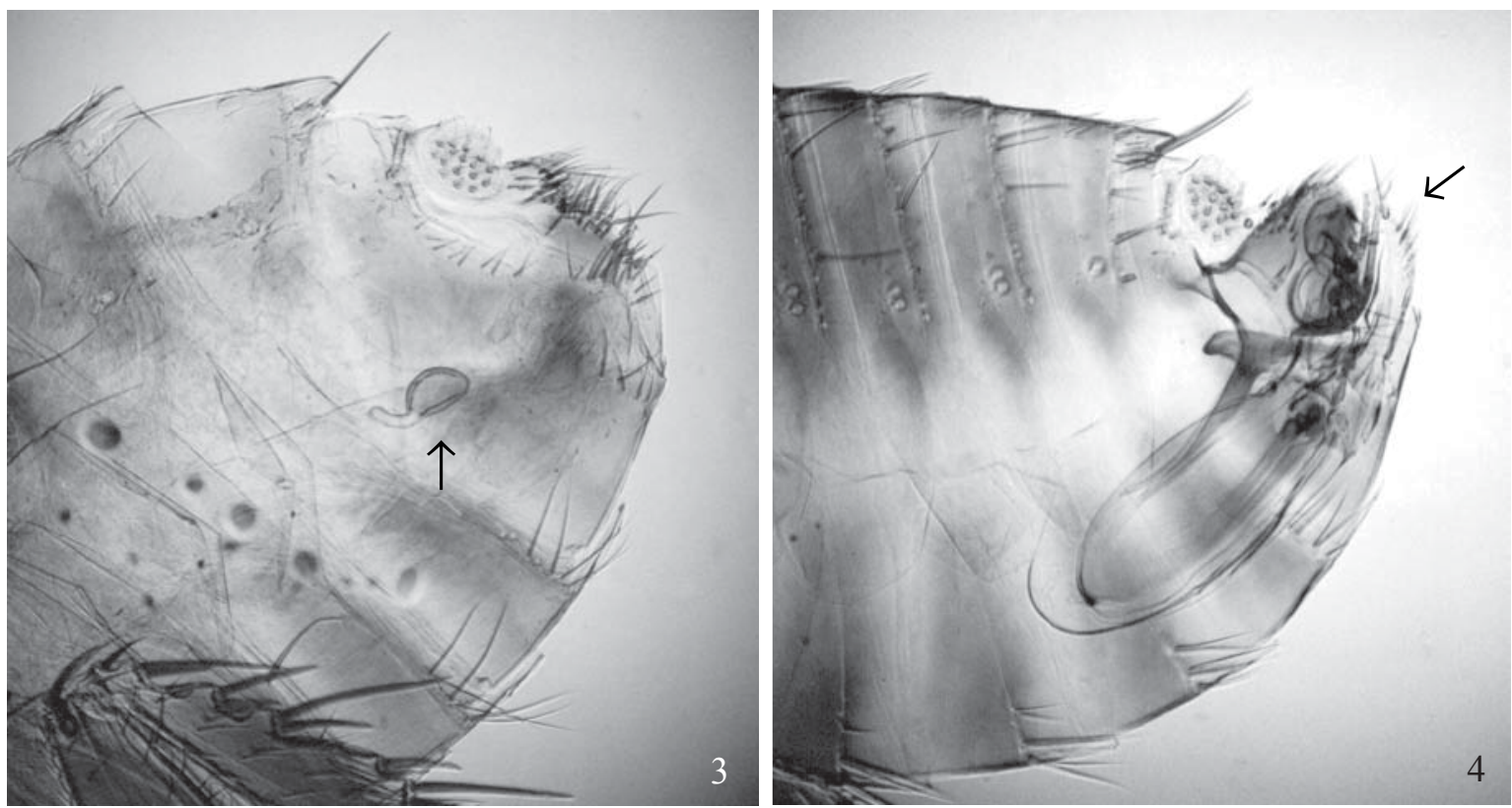

Figs. 3-4. Rhopalopsyllus lutzi lutzi. 3. Genitália da fêmea apresentando espermateca com bulga elíptica; 4. Genitália do macho com segmentos modificados: têlomero e esternito IX.

solitaria e N. socialis, ambos sinonímias de Nasua nasua Linnaeus, 1776) por Linardi \& Guimarães (2000).

No presente trabalho, $R$. lutzi lutzi é, pela primeira vez, assinalada em oito cães domésticos (Canis familiaris) naturalmente infestados em áreas rurais do município de Piraí, estado do Rio de Janeiro. Os dados obtidos ampliam a faixa de hospedeiros conhecidos, salientando para a importância dos hábitos predatórios de hospedeiros carnívoros na disseminação da referida espécie de sifonáptero. Observações dessa natureza deveriam estender-se para outras regiões do país, tendo em vista estudos concernentes a impactos ambientais e expansão do ambiente urbano sobre o ambiente silvestre. Ressalte-se ainda, o significado epidemiológico dos Rhopalopsyllidae na veiculação de etiologias entre animais silvestres e destes para animais domésticos.

\section{REFERÊNCIAS}

Barros, D. M.; P. M Linardi \& J. R. Botelho.1993. Ectoparasites of some wild rodents from Paraná State, Brazil. Journal of Medical Entomology 30: 1068-1070.

Barros-Battesti, D. M. \& M. Arzua. 1997. Geographical distribution by biomes of some Marsupial Siphonaptera from the State of Paraná, Brazil. Memórias Instituto Oswaldo Cruz 92: 485-486.

Beauty, B. J. \& W. C. Marquardt. 1996. II. Fleas and the agents they transmit, p. 146-159. In: R. E. Thomas (ed.).The Biology of Disease Vectors. University Press of Colorado, p. 632.

Bicho, C. L. \& P. B. Ribeiro. 1998. Chave pictórica para as principais espécies de Siphonaptera de importância médica e veterinária, no Brasil. Revista Brasileira de Parasitologia Veterinária 7: 4751 .
Jordan, K. \& N. C. Rothschild. 1923. On the genera Rhopalopsyllus and Parapsyllus. Ectoparasites 1: 320-370.

Kettle, D. S. 1995. Siphonaptera (Fleas), p.323-343. In: D. S. Ketle (ed.). Medical and Veterinary Entomology. Cambridge University Press, p. 485.

Linardi, P. M. 1984. Relações taxonômicas e filogenéticas entre os gêneros de sifonápteros Ropalopsilinos obtidas do estudo das relações hospedeiro/parasito. Revista Brasileira de Biologia 44: 329-334.

Linardi, P. M. 1985. Dados complementares sobre hospedeiros de sifonápteros Ropalopsilinos. Revista Brasileira de Biologia 45: 73-78.

Linardi, P. M. 1987. Distribuição geográfica dos sifonápteros Ropalopsilinos. Revista Brasileira de Biologia 47: 385-396.

Linardi, P. M. \& L. R. Guimarães. 1993. Systematic review of genera and subgenera of Rhopalopsyllinae (Siphonaptera: Rhopalopsyllidae) by phenetic and cladistic methods. Journal of Medical Entomology 32: 161-170.

Linardi, P. M. \& L. R. Guimarães. 2000. Sifonápteros do Brasil. São Paulo, Ed. Museu de Zoologia USP/FAPESP, $291 \mathrm{p}$.

Scofield, A.; M. F. Riera; C. Elisei \& C. L. Massard. 2002. Ocorrência de sifonápteros dos gêneros Polygenis (Rhopalopsyllidae) e Ctenocephalides (Pulicidae) em cães procedentes de zona rural, município de Piraí, estado do Rio de Janeiro. A Hora Veterinária 126: $18-20$

Smit, F. G. A. M. 1987. An Illustrated Catalogue of the Rothschild Collection of Fleas (Siphonaptera) in the British Museum (Natural History). Vol. VII Malacopsylloidea. Oxford \& London, Oxford University Press, $380 \mathrm{p}$.

Soulsby, E. J. L. 1987. Artrópodos, p. 356-510. In: E. J. L. Soulsby. Parasitología y Enfermedades Parasitarias en los Animales Domésticos. Mexico, D. F., Nueva Editorial Interamericana S. A. de C. V., 824 p.

Wilson, D. E. \& D. M. Reeder. 1993. Mammal Species of the World. Washington, D. C., Smithsonian Institution Press. 1206 p. (também disponível na Web: www.nmnh.si.edu/msw/). 